\title{
THE EFFECT OF HYDROGEN CHLORIDE ON THE NITROGEN-HYDROGEN EQUILIBRIUM.
}

\author{
By E. B. LUDLAM.
}

(A Pafer commulicated to the Transactons of THE FARADAY Society. Receiced February 1917.)

The equilibrium existing between nitrogen, hydrogen, and ammonia has been investigated at temperatures from $500^{\circ} \mathrm{C}$. to $\mathrm{r}, 000^{\circ} \mathrm{C}$. by $\mathrm{Haber}$, and his work laid the foundations of the manufacturing process which has since been developed. For its success this process depends upon high pressure, purity of materixls, and efficacy of catalysts. Any process which could dispense with some or all of these difficult conditions might be expected to prove itself a serious rival.

The early experiments of Deville all pointed to the probability that ammonia in the presence of hydrochloric acid gas was far more stable than when alone. Thus, at $920^{\circ} \mathrm{C}$., the boiling-point of zinc, his results for the vapour density of ammonium chloride indicate that about is per cent. of the ammonium chloride is not even dissociated into ammonia and hydrogen chloride, still less is any of the ammonia decomposed into nitrogen and hydrogen.

Further, he heated two tubes side by side in the same furnace ; through one he passed ammonia and in the other he heated ammonium chloride. Although the ammonium was almost completely decomposed, the ammonium chloride showed hardly any decomposition unless the temperature was over $\mathrm{I}, 000^{\circ} \mathrm{C}$.

He also passed the three gases through a hot porcelain tube, and obtained a deposit of ammonium chloride on a water-cooled tube placed along the centre of the porcelain tube.

On sparking a mixture of nitrogen and hydrogen, he obtained only traces of ammonia, but in presence of hydrogen chloride combination was complete.

Extraordinary as it may appear, no really careful revision of this work seems to have been carried out.

By a combination of the results obtained by Haber and by Deville, it would appear reasonably probable that the addition of hydrochloric acid to nitrogen and hydrogen would displace the equilibrium largely in the direction of the formation of ammonia by preventing the reverse change. It would then be no longer necessary to rely on high pressure, for high temperature would secure a sufficiently rapid rate of formation, and, for the same reason, the exact nature of the catalyst would become a matter of smaller importance. In short, a temperature of about $\mathrm{r}, \mathrm{OoO}^{\circ} \mathrm{C}$. might be made to produce a rate of change comparable to that obtained at lower temperatures by means of pressure and carefully selected catalysts, but the yield of ammonia would be high on account of the conserving action of the hydrochloric acid. It was not to be expected that the catalysts which had 
been found most suitable by Haber would be available when hydrochloric acid was employed.

On the other hand, a process was patented in 1880 by Glover in which producer gas was mixed with hydrochloric acid gas and then passed over red-hot coke, but nothing more seems to have been heard of the process. Mond, also, appears to have organized similar experiments, and to have come to the conclusion that no ammonia was produced which was not fully accounted for by the nitrogen known to have been present in the coke. More recently, in 1905, M. Berthelot carried out a series of experiments in which ammonium chloride was heated in quartz tubes at $\mathrm{r}, 300^{\circ} \mathrm{C}$. He found complete decomposition in all cases, but he also found that the hydrogen diffused away, so the experiments were not very conclusive and were of little value as evidence as to the state of equilibrium in the neighbourhood of $\mathrm{I}, 00 \mathrm{O}^{\circ} \mathrm{C}$.

The matter seemed worthy of investigation, and preliminary experiments were commenced, but the more carefully these were performed, and the greater the care expended in purifying the materials, the smaller was the quantity of ammonia produced. This led to some doubt of Deville's results, and experiments were started to test their validity. In one of these, ammonium chloride vapour was passed backwards and forwards through a quartz tube containing broken quartz, any uncondensed gas being swept into a nitrometer by means. of carbon dioxide. It was found that below $900^{\circ} \mathrm{C}$. there was very little decomposition, but at $\mathrm{I}, 000^{\circ} \mathrm{C}$. considerable quantities of nitrogen and hydrogen were formed.

It was clearly worth while to push the inquiry a little further, even though it now appeared more likely than not that the original premises were false.

It is rather remarkable that the data for the behaviour of ammonium chloride are very incomplete for temperatures above $300^{\circ} \mathrm{C}$, considering how much study has been devoted to the vapour density of this substance. Very recently some excellent work on the subject has been carried out by Professor Alexander Smith and his pupils in America. He states: "In the case of solids undergoing thermal dissociation the data that possess the greatest chemical significance are those relating to the saturated vapours, in which the components of the vapour are in equilibrium with each other and with the solid. Yet in regard to ammonium chloride quantitative information in this direction is absolutely lacking. The vapour density has been determined repeatedly, but for the most part the densities of the unsaturated vapours have alone been measured. It is commonly assumed that the dissociation is complete, and the densities correspond with this assumption. The results of this present work show it to be very far from correct."

From Professor Smith's work it is now possible to caluulate the dissociation constants, the degree of dissociation, the partial pressures of the products, the heats of dissociation and the latent heats of vaporization over a range of temperature up to $380^{\circ} \mathrm{C}$. But the gap between this and $920^{\circ} \mathrm{C}$. is so prodigious that extrapolation would be futile. However, he found that at . $330^{\circ} \mathrm{C}$., for example, ammonium chloride was only 63 per cent. dissociated and the vapour pressure was $610 \mathrm{~mm}$. His figures show that with increase of temperature the dissociation of the saturated vapour decreases. This is explained by the rapid increase in the vapour pressure, which reaches I,063 mm. at $35^{\circ} \mathrm{C}$. Further, the heat absorbed by dissociation at these temperatures is about $40,0 n 0$ calories, and increases with rise of temperature.

The heat of formation of ammonium chloride from nitrogen, hydrogen and hydrogen chloride is given at about 70,000 calories. Compared with 
this, the heat of formation of ammonia from nitrogen and hydrogen is small, 12,200 calories. This points to the probability that the economical maintenance of a high temperature for the synthesis would be an easy matter. In Haber's process there is even some difficulty in preventing the temperature rising too high, locally, owing to the heat of formation of the ammonia.

It is worthy of note that in the Haber reaction the ratio of the volumes before and after is as two to one. For the formation of ammonium chloride it is as three to two, if the substance is fully dissociated, and three to one if undissociated. If the ammonium chloride is condensed in situ, the volume reduction would be practically to zero, as, for example, in any process based on the "hot and cold tube" principle.

As the immediate object of the work was to decide on the possibility of effecting a commercial synthesis, the first experiments performed were based on the principle of Deville's hot and cold tube experiment. The apparatus was of an extremely simple type (see Fig. I). Equivalent volumes of nitrogen, hydrogen, and hydrogen chloride were passed through the tube, the imer-

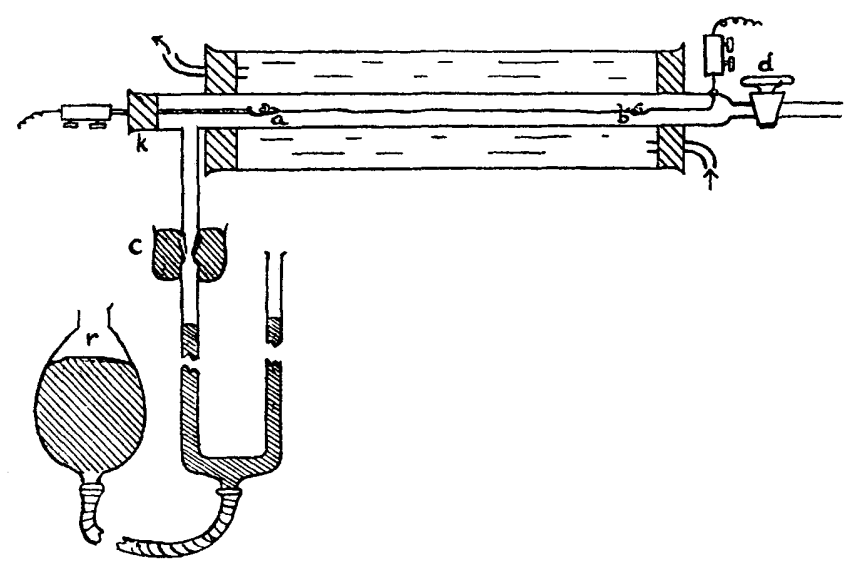

FIG. 1.

cury receiver, $r$, being lowered so that there was a free passage for the gas through the manometer. The mercury was then raised to a level just below the mercury cup, $c$, round the ground-glass joint and the tap, $d$, was closed. The heating was performed by passing an electric current through the wire, $a b$, which became the hot surface. The current was led through the cemented cork, $k$, by a thick silver wire and at the other end through the glass by stout platinum wire. A constant stream of cold water circulated through the outer jacket.

On switching on the current, the wire, $a b$, was raised to a just perceptibly dull red heat. This was accompanied by an immediate expansion of the gas, shown by the depression of the mercury in the manometer, but in a minute or two the volume became practically constant. Convection currents streamed upwards from the wire, downwards round the cold surface and vertically up again to the wire.

The temperature could be maintained constant for many hours by maintaining the current constant. It could then be increased and again left, raised again and so on, until a range of temperature from $500^{\circ} \mathrm{C}$. to $1,500^{\circ} \mathrm{C}$. had been covered. 
Platinum wire was the first to be used. Formation of ammonium chloride and its condensation on the cold surface would have been accompanied by a steady diminution in volume, but no such effect was noticed. On finally cooling, washing out the tube and testing with Nessler's solution, there was only a trace of ammonia found.

A carbon rod was next employed, made by the Ediswan Company, with platinum wires attached by means of "paste." Even when the experiments were extended over 24 hours there was no evidence of the continuous formation of ammonia, and Nessler's solution again showed only a trace at the conclusion of the experiment.

Experiments without hydrochloric acid showed no appreciable difference.

The experiment was then varied by soaking the carbon rot in solutions of common salt, calcium chloride, and magnesium chloride respectively. There was no increase in the amount of ammonia produced; the only difference noticeable was that these substances were volatilized at the temperature of a bright red heat and deposited on the cold surface.

The carbon was next electroplated with a thin film of copper. At a dull red heat cuprous chloride volatilized, but again no increased quantity of ammonia was obtained.

A spiral of iron wire was wrapped round a thin quartz rod. At a temperature near, but below, a red heat, the iron suddenly formed white fumes and the temperature rose quickly to a red heat. The white fumes travelled in clearly visible convection currents to the cold surface, forming rings round the glass tube corresponding to the loops of the spiral. This white sublimate was found to be ferrous chloride. It contained no ammonia.

So far, then, as these experiments go, they indicate no advantage of the kind deemed possible as the result of the presence of hydrochloric acid. The hot surface is certainly very small, but on the other hand, the time could be extended indefinitely ; whereas the small quantity of gas and the simplicity of the apparatus were very considerable advantages. The greatest pains were taken with experiments between $800^{\circ} \mathrm{C}$. and $\mathrm{r}, 000^{\circ} \mathrm{C}$.; subsequent experiments suggest that it might have been better to have concentrated attention more particularly on temperatures in the neighbourhood of $1,100^{\circ} \mathrm{C}$., but at the time there was no indication of more ammonia being formed at these than at lower temperatures.

The next experiment was a careful attempt to compare the amounts of ammonia formed with and without the presence of hydrochloric acid, other conditions being identical.

The method chosen was to take two similar quartz tubes, place them side by side in the same furnace, pass the mixture of nitrogen and hydrogen through one, absorb the ammonia, then add the hydrochloric acid and pass the gases through the other tube, collecting the ammonium chloride on a water-cooled silver tube. The temperature chosen was $800^{\circ} \mathrm{C}$, , the tubes were filled with pure sugar charcoal by way of catalyst and the gases were passed extremely slowly ( 16 litres in 48 hours).

Fig. 2 indicates diagrammatically the arrangement of the apparatus. The nitrogen and hydrogen were contained under pressure in a gas cylinder provided with a pressure gauge and sensitive gas regulator. The actual proportions were 45 of nitrogen to 55 of hydrogen. They passed first over heated palladized asbestos to ensure the removal of all oxygen. No rubber connections were made, but mercury cups were found very convenient ; they are indicated in the diagram by $c_{1} c_{2} c_{3} c_{4} c_{5} r_{6}$. The rate of passage was determined by counting the bubbles per minute through the potash bulb, $h$, after which the gases were dried by the long calcium chloride tube, $d$, and passed 


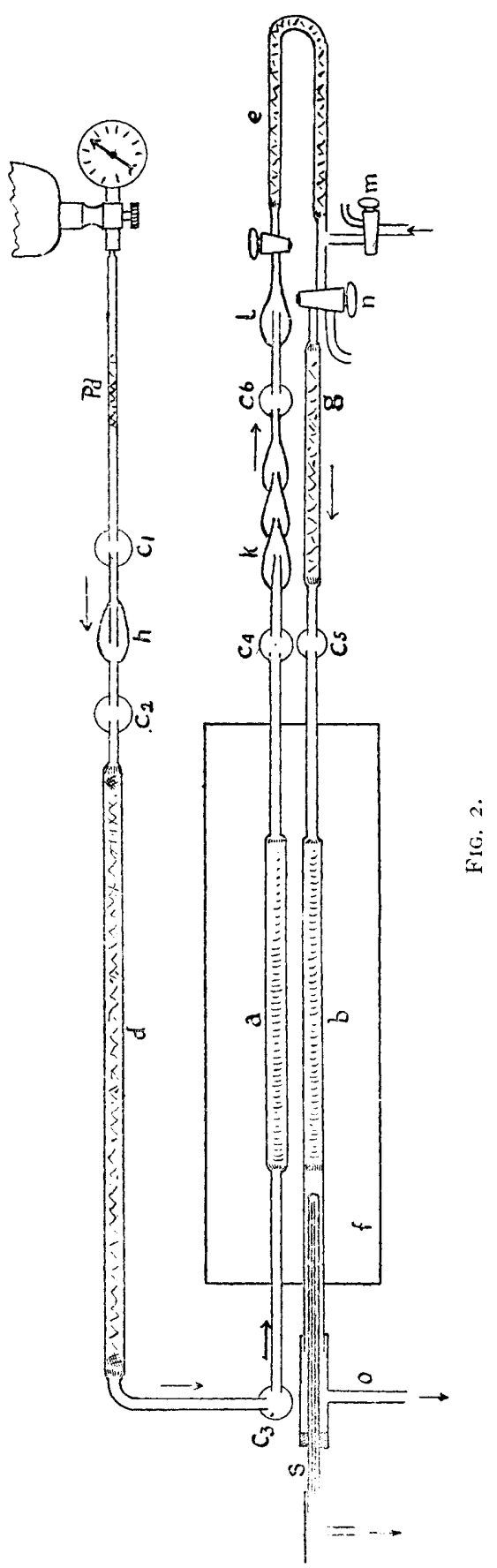


into the furnace through the tube, $a$, and thence through the bulbs, $k$, containing dilute acid to absorb the ammonia. They then passed through a potash bulb, $l$, were dried in a calcium chloride tube, $o$, received the hydrochloric acid from the three-way tap, $n$, traversed another calcium chloride tube, $g$, and so into the quartz lube lying alongside the tube $a$. At the other end of this tube, still in the hot part of the furnace, a silver tube was placed, through which a stream of cold water was circulated. The exit was closed from the atmosphere by mercury first, and then by water, through which the gases bubbled.

It was not found easy to regulate the supply of hydrochloric acid with any exactness. Samples of the gas could be withdrawn at the three-way tap, $n$, and the proportion of hydrochloric acid varied from 3 to 5 per cent. After trying several methods, it was found to be much the best to use a Kipp's apparatus, containing ammonium chloride and concentrated sulphuric acid. The gas was washed by bubbling through three bulbs containing

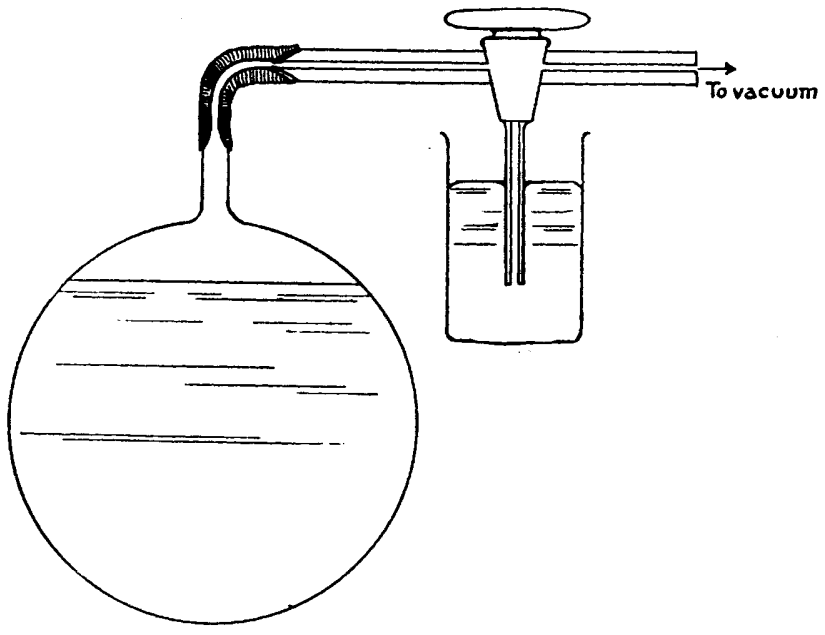

FIG. 3.

calcium chloride solution and dried by passage over calcium chloride. This method was not used before drastic tests had been performed to ascertain whether any traces of ammonium compounds were carried over and no trace found.

At the conclusion of the experiment the washings of the exit tubes and of the silver tubes were found to contain only very small quantity of ammonia, too little for estimation by alkalimetry ; similarly in the bulbs containing acid for the absorption of ammonia. Accordingly, the animonia was estimated with Nessler's solution by comparison with standard dilute ammonium chloride solution. The ammonia formed in the presence of hydrochloric acid was $3^{\prime 2}$ times as much as in the case of the nitrogen alone, but even so, was only one seven hundredth of the amount calculable from Haber's results for $\mathrm{K}$ in the equation

$$
\mathrm{K}_{力}=\frac{\left[\mathrm{N} \mathrm{H}_{3}\right]}{[\mathrm{N}]^{\frac{1}{2}} \times[\mathrm{H}]^{3}}
$$

This means that the reaction was procceding at a rate so extremely slow that there was no approach to equilibrium. It showed that the problem of 
finding a catalyst suitable for use in the presence of hydrochloric acid was likely to prove an even greater difficulty than had been anticipated. Each experiment with a different catalyst would consume much time, for only experiments carried out with the utmost care would be of any use.

It was therefore decided that the desired information would be obtained more rapidly and certainly by approaching the equilibrium from the other side, starting from ammonium chloride and decomposing it into nitrogen, hydrogen, and hydrochloric acid. For this the simplest apparatus was all that was necessary. A quartz bulb was employed of about 200 c.c. capacity, provided with a narrow stem which cosld be sealed off in the oxy-hydrogen blowpipe.

In this bulb a weighed quantity of ammonium chloride was placed, sufficient to produce in the evacuated bulb a pressure roughly approximate to that of the atmosphere, at the temperature to which the bulb was to be

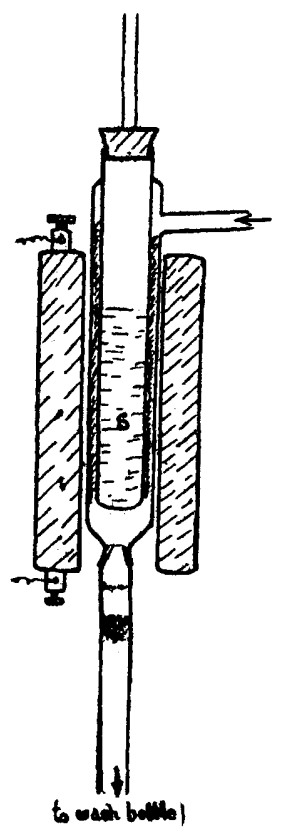

FIG. 4 .

heated. It was impossible to tell, a priori, what the exact pressure would be, for that would have involved a knowledge of the result which the experiment was designed to ascertaiı. Before sealing off, the bulb was washed out twice with nitrogen, using a Geryk pump, and then evacuated to a pressure of about $1 \mathrm{~mm}$. of mercury. It was then placed in an electric furnace and maintained at the requisite temperature for a convenient length of time. On removal from the furnace it was cooled rapidly in running water or in a bowl of mercury. On breaking open the tube under mercury, the latter entered until the pressure inside was atmospheric ; a rubber tube closed by a threeway tap was then slipped over the end and the gas sucked out, by means of the arrangement shown in Fig. 3 , into a gas analysis apparatus. Absorption by water gave the volume of hydrochlorie acid remaining, and this could be 
checked by titrating the solution with standard dilute sodium carbonate solution. The hydrogen was determined by adding oxygen, and noting the reduction in volume on passing backwards and forwards over hot palladized asbestos.

\section{SUMmaRY OF RESULTS.}

(a) Ammonium chloride was heated alone for 5 hours at $95^{\circ} \mathrm{C}$. There was no residual gas except the small quantity of nitrogen left by the pump. There was no contraction after mixing with oxygen and passing over hot palladized asbestos. Conclusion : Practically no decomposition. As Deville's experiments on the vapour density of ammonium chloride did not last half an hour, the above experiment fully supports his result.

(b) It was impossible to heat the bulb very rapidly up to the desired temperature, as a certain amount of time is consumed before the gaseous contents can have attained the temperature of the furnace. The bulb was put into the cold furnace and the temperature raised in the course of an hour to $900^{\circ} \mathrm{C}$., an hour later it had risen to $\mathrm{x}, 000^{\circ} \mathrm{C}$., and two hours later still to $\mathrm{I}, 100^{\circ} \mathrm{C}$., when an internal contact in the furnace gave way. The residual gas contained 2 c.c. of hydrogen, indicating decomposition of to per cent.

(c) In the next experiment, precipitated gold was added (M.P. $1,063^{\circ} \mathrm{C}$.). The ammonium chloride was heated for 3 hours at $\mathrm{I}, 000^{\circ} \mathrm{C}$., and on rapid cooling it was evident from the amount of solid ammonium chloride visible (cubical crystals) that there had been very little decomposition. The bulb was not opened, but was heated again for 5 hours from $\mathrm{I}, 000^{\circ} \mathrm{C}$. to $\mathrm{I}, 05^{\circ} \mathrm{C}$. The residual gas contained $8 *_{4}$ c.c. of hydrogen, indicating 32 per cent. decomposition.

(d) In this experiment, precipitated silver was used, to see whether it would facilitate the decomposition at a lower temperature (M.P. $96 \mathrm{I}^{\circ} \mathrm{C}$.). The temperature was kept in the neighbourhood of $950^{\circ} \mathrm{C}$. for 4 hours. Only I C.C of hydrogen was found in the residual gas, equivalent to $4^{\circ} 6$ per cent. decomposition.

(e) A silver mirror was deposited on the inside of the bulb, covering nearly the whole surface. To avoid any risk of melting the silver, the temperature was only taken to $900^{\circ} \mathrm{C}$., but was kept there for 7 hours. The decomposition was only 2.3 per cent. The effect of the heating had been to destroy the mirror and convert it into a thin grey film.

$(f)$ Copper was next tried, about half a metre of fine copper wire being used. This experiment was spoilt owing to a defective contact in the millivoltmeter used in conjunction with the thermo-couple. In consequence the temperature rose to about $\mathrm{I}, 200^{\circ} \mathrm{C}$., instead of remaining constant at $950^{\circ} \mathrm{C}$. It was impossible to judge how long the temperature had been rising. The copper had melted. Incidentally the pressure must have greatly exceeded that of the atmosphere. The residual gas showed that the decomposition was about 70 per cent.

(g) It was decided to try iron, which is known to be a good catalyst for nitrogen and hydrogen alone. The action of the hydrochloric acid on the iron introduced a factor, however, which would make the results somewhat ambiguous. As the apparatus did not lend itself conveniently to the reduction of metals in situ, about half a metre of fine Swedish charcoal iron wire was placed in the bulb with 0.378 gramme of ammonium chloride. This was left for 5 hours at $800^{\circ} \mathrm{C}$., and then allowed to remain all night, the temperature falling very slowly so that it had reached $700^{\circ} \mathrm{C}$. 15 hours later. On opening under water the total volume of gas obtained was $39^{\circ} 9$ c.c. $\left(15^{\circ} \mathrm{C}\right.$. and $760 \mathrm{~mm}$.). The analysis showed $29^{\circ} 9$ c.c. hydrogen and 100 c.c. nitrogen. The 
free hydrochloric acid in solution neutralized 3 c.c. of twentieth normal sodium carbonate solution equivalent to 3.5 c.c. hydrochloric acid gas $\left(a t 15^{\circ} \mathrm{C}\right.$. and $760 \mathrm{~mm}$.). More sodium carbonate precipitated the iron in solution, the greenish white precipitate at first formed turning rapidly brown. The clear liquid was tested with Nessler's solution, and only a trace of ammonia was present. The decomposition was practically complete. Much more exact methods would be necessary in order to compare the small amount of ammonia remaining with the equilibrium quantity for nitrogen and hydrogen alone. The setting up of a new equilibrium between the iron, the hydrogen chloride, the hydrogen and the ferrous chloride, should give rise to a proportion of hydrogen to nitrogen greater than 3 to $I$, whereas the analysis showed it to be almost exactly $3: \mathrm{I}$. For purely scientific interest this experiment, or a modification of it, deserves repetition and exact study, but for the present purpose it seemed to indicate sufficiently clearly that the final equilibrium at $800^{\circ} \mathrm{C}$. was not displaced to any very considerable extent in the direction of the formation of ammonia by the presence of hydrochloric acid. At any rate, it seemed more important to proceed to experiments at lower temperatures in the presence of so good a catalyst as iron. This was abandoning altogether the original conception based on Deville's work, now that it appeared practically certain that ammonium chloride is not really stable at high temperatures, but decomposes with extreme slowness.

(h) An experiment similar to the last was carried out at $500^{\circ} \mathrm{C}$. instead of $800^{\circ} \mathrm{C}$. After heating for 3 hours the bulb was opened under water. The solution tested with methyl orange was entirely free from acid; on standing it turned brown and a precipitate formed. The clear solution gave no further precipitate on adding sodium hydroxide, but on adding Nessler's solution a very dense precipitate was obtained. The gas left in the bulb consisted of 3.4 c.c. of hydrogen and $1 \cdot 2$ c.c. of nitrogen. Here again there is a deficiency in the hydrogen not easily explained. Calculating on the basis of the total nitrogen found as gas the decomposition was about I 2 per cent.

Another experiment carried out in exactly the same manner, but for I 8 hours, gave $3 \mathrm{I}$ c.c. of nitrogen and hydrogen. An analysis showed the ratio of $\mathrm{N}: \mathrm{H}$ to be $\mathrm{I}: 2.5$. The proportion of hydrogen is still lower than in the previous experiment. Calculating from the total gas, the decomposition was 53 per cent. By way of checking this figure, the solution was distilled with sodiun hydroxide, and the ammonia passed into standard acid and titrated, with methyl orange as indicator. It was found that ' 026 gramme of ammonium chloride remained out of 0512 gramme, a decomposition of $4^{8}$ per cent. Hence both results agree in making the decomposition approximately 50 per cent.

Without spending further time on refinements to secure more accurate results, it seemed desirable to examine whether any synthesis could be effected by the use of iron at these low temperatures.

In a preliminary experiment, an iron tube was filled with asbestos which had been soaked in ferrous chloride and dried. The tube was heated in an electric furnace to $500^{\circ} \mathrm{C}$, and hydrogen was passed through to reduce the ferrous chloride to iron. Then nitrogen and dry hydrochloric acid gas were also passed through. A white sublimate was obtained which easily re-sublimed, and a trace of it gave an intense colour with Nessler's solution. A little of it moistened with sodium hydroxide solution gave a smell of ammonia.

As the apparatus did not lend itself to quantitative work, a piece of quartz apparatus which had been used for another purpose was turned to account. It consisted of two tubes fused together at one end and separated by an 
annular space. The inner tube was closed inside; the outer tube was open to admit gas at one end, and was provided with a ground quartz joint at the other (see Fig. 4). The tube was placed in an electric furnace, and sulphur was placed in the central tube. The electric current could be carefully adjusted so that the sulphur was kept just steadily boiling. The space between the two tubes was packed with iron asbestos. As the space was very narrow the temperature gradient across it would be only slight, and the temperature of the iron asbestos could be taken as $450^{\circ} \mathrm{C}$. Hydrogen alone was first passed, and then the three gases in the proportions nitrogen to hydrogen as $I: 3$, with the addition of about io per cent. hydrochloric acid. It was soon noticed that two sublimates were formed, one very slight, just at the exit of the furnace, the other forming in larger amount about a centimetre lower down, The first was ferrous chloride, the second ammonium chloride.

With the object of carrying out a careful quantitative comparison between the amount of ammonia produced with and without hydrochloric acid, nitrogen and hydrogen in equivalent proportions were passed for 22 hours at the rate of 800 c.c. per hour ; the issuing gases were passed through a washbottle containing dilute sulphuric acid, but the total volume of ammonia produced from 17.8 litres of the gases was only $2 \cdot 1$ c.c.

Repeating now with the addition of ro per cent. of hydrochloric acid, the ammonium chloride collecting in the quartz tube was washed out and added to any that might have been carried forward into the washbottle and distilled from caustic soda into standard acid. Calculated as ammonia gas only 2.4 c.c. were obtained. This result is slightly better than without hydrochloric acid, but in both cases the quantity is very minute. On removing the tube from the furnace it was noticed that the asbestos, which originally had been dark grey, was now nearly white ; it was evident that the iron had nearly all volatilized as ferrous chloride.

Thus, even at a temperature so low as $45^{\circ} \mathrm{C}$. the volatility of ferrous chloride is a serious obstacle to encounter, even if the method should be found free from other objections. It is hardly to be expected that the formation of ammonia at atmospheric pressure would proceed at a useful rate at any temperature much lower. 